\title{
Design, Dynamic Analysis and Optimization of a Rover for Rescue Operations
}

\author{
Hadi Tavakoli Nia ${ }^{1}$, Seyed Hamidreza Alemohammad ${ }^{1}$, Saeed Bagheri ${ }^{1}$, \\ Reza Hajiaghaee Khiabani ${ }^{1}$, and Ali Meghdari ${ }^{2}$ \\ Center of Excellence in Design, Robotics and Automation \\ Department of Mechanical Engineering \\ Sharif University of Technology, P.O. Box: 11365-9567, Tehran, Iran \\ meghdariesharif.edu
}

\begin{abstract}
In this paper a new approach to dynamic optimization of a rough terrain rover is introduced. Since rover wheels traction has a significant role in rover mobility, optimization is based on the minimization of traction at rover wheel-ground interfaces. The method of optimization chosen is Genetic Algorithm (GA) which is a directed random search technique along with the usual optimization based on directional derivatives. GA is a suitable and efficient method of optimization for nonlinear problems. The procedure is applied on a specific rough terrain rover called CEDRA-I Shrimp Rover. The present work resulted in design and manufacturing of the optimized rover called CEDRA-II Shrimp Rover.
\end{abstract}

\section{Introduction}

Rough terrain rovers are increasingly used for high risk situations such as rescue operations, planetary explorations and military missions. Future tasks will require robots with high mobility. Keeping stability without tip over or loss of contact with the ground is needed for those hazardous tasks.

In these cases having a flexible rover that can adapt itself to environment of challenging tasks, is very useful. Robots with passively articulated suspensions can improve rough-terrain mobility by modifying their suspension configuration and thus repositioning their center of mass.

Design and control of these rovers are based on dynamical analysis and simpler and more meaningful equations of motion may be helpful in many cases. The complication of equations of motion in rovers arises from several factors including: mechanism with complicated configuration, rough terrain usually with random bumps and nonholonomic constraints.

Much research has been done on rough terrain rovers dynamical analysis and modeling. Tai [1] presented dynamical modeling for mobile robots with suspension. Iagnemma and Dubowsky [2] presented a new method for rough terrain rovers'

\footnotetext{
${ }^{1}$ Graduate Student.

${ }^{2}$ Professor of Mechanical Engineering.
} 
control. Their work is based on the static modeling of rovers due to the rovers low speed.

Kawabe et al. [3] performed substantial work on traction control of passenger vehicles on flat roads. This work is not applicable to low-speed, rough terrain rovers because in these vehicles wheel slip is caused primarily by kinematic incompatibilities. Reister and Unseren [4] studied the traction control for low-speed mobile robots on flat terrains. Sreenivasan and Waldron [5] have represented the displacement analysis of articulated wheeled vehicle configuration and have extended it to uneven terrain motion. Hacot [6] illustrated the traction control of a planetary rover called Rocker Bogie. In Ref. [7], optimization of a four-wheel rover is presented. The optimization objective function is finding the rover parameters so that the path traversed by the center of gravity tends to the straight line.

In this paper an innovative approach to dynamical optimization of a rough terrain rover with redundant drive wheels is presented. Optimization is based on the minimization of traction in the rovers' wheel-ground contact. The method of optimization is chosen to be Genetic Algorithm (GA) which is known as a directed random search technique. GA is a suitable and efficient method of optimization for nonlinear problems. In this paper dynamical equations are developed using Kane's method. Compared to other formulations, (Lagrange or Newton) Kane's method involves less arithmetic operations. So, the model simulation is fast and simple. Also, Kane's equations can be easily brought into closed form [8].

Finally the analysis is applied to CEDRA-I Shrimp rover, which is a complicated rough terrain rover with six-wheels. CEDRA-I Shrimp rover is a laboratory rover made at the Center of Excellence in Design Robotics and Automation (CEDRA) and ranked second in the Rescue Robot Competitions 2003 (RoboCup Rescue 2003). The main structure is based on the rover, which first was constructed at EPFL [9]. This robot is similar to Rocky7 [10] and Marsokhod [11] in some parts but a four-link mechanism added at the front of the robot has made it more efficient in encountering obstacles. The present work resulted in design and manufacturing of the optimized rover called CEDRA-II Shrimp rove. Both initial and the optimized rovers are shown in Fig. 1.

\section{Dynamics Equations}

\subsection{Kinematic Analysis}

The kinematic analysis is the base point in dynamics analysis and as a result the optimization. Analyses which have been done on rovers up to now are usually for surfaces with simple and specified geometry like an inclined surface; however for a rough terrain, the rover kinematical problem will change significantly. Rover kinematical analysis of complex geometrical surfaces leads to several nonlinear equations; the solution to these problems is the most time consuming part of the analysis. In this section the planar inverse kinematical analysis of the shrimp mechanism is dealt as an example. 


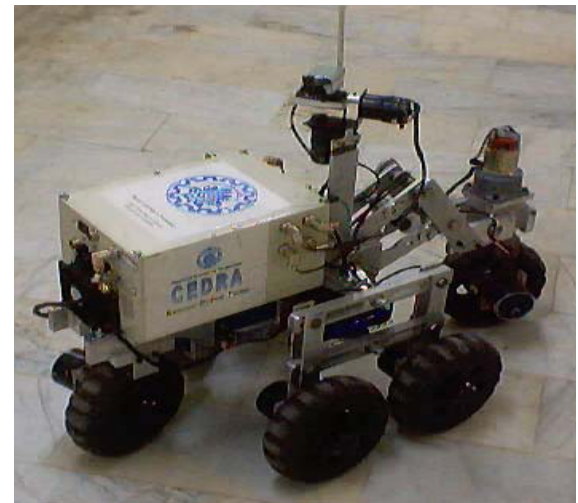

(a)

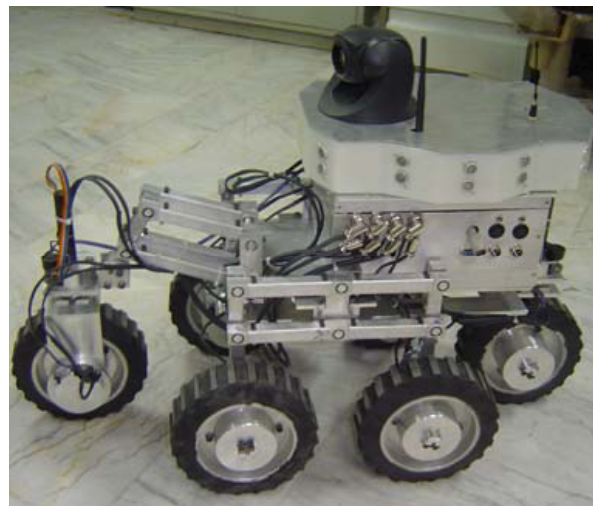

(b)

Fig. 1. (a) CEDRA-I and (b) CEDRA-II rescue robots

The shrimp mechanism (see Fig. 2) is a one DOF mechanism. Having the path geometry in hand, one can determine the position of all mechanisms linkages, using the position of the rear wheel. In general for a rover with $n$ wheel-ground interface points one can obtain $n-1$ close kinematic loop. In the Shrimp mechanism for interface points $\mathrm{B}, \mathrm{C}$ and $\mathrm{D}$ one can write:

$$
\begin{gathered}
B_{x}=c \sin \alpha+e \cos (\alpha+\beta) \\
B_{y}=-c \cos \alpha+e \sin (\alpha+\beta) \\
C_{x}=c \cos \alpha-e \sin (\alpha+\beta) \\
C_{y}=-c \sin \alpha-e \cos (\alpha+\beta) \\
D_{x}=(a+b-g) \cos \alpha+f \sin \alpha+n \cos \gamma+m \cos \xi \\
D_{y}=(a+b-g) \sin \alpha-f \cos \alpha+n \sin \gamma+m \sin \xi
\end{gathered}
$$

where

$$
\begin{aligned}
\xi= & \gamma-\cos ^{-1}\left(\frac{n-h \cos (\pi / 2-\gamma+\varphi)}{h^{2}+n^{2}-2 n h \cos (\pi / 2-\gamma+\varphi)}\right) \\
& -\cos ^{-1}\left(\frac{h^{2}+n^{2}-2 n h \cos (\pi / 2-\gamma+\varphi)+l^{2}-k^{2}}{2 l \sqrt{h^{2}+n^{2}-2 n h \cos (\pi / 2-\gamma+\varphi)}}\right)
\end{aligned}
$$

Angles $\alpha, \beta$ are shown in Fig. 2 . 

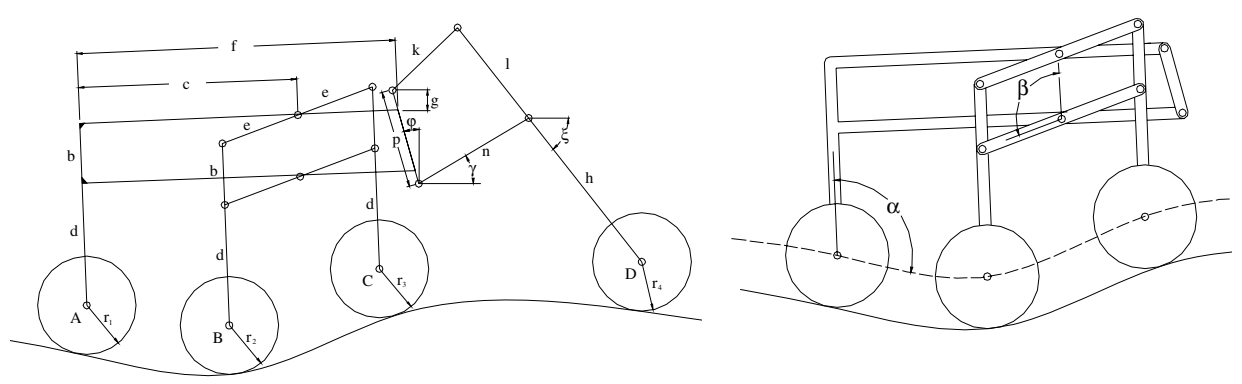

Fig. 2. Shrimp rover mechanism

For simplicity, the wheels center line path is considered rather than the real path. If the wheel center line path's function is shown as $y=\operatorname{path}(x)$, then

$$
\begin{aligned}
& B_{y}=\operatorname{path}\left(B_{x}\right) \\
& C_{y}=\operatorname{path}\left(C_{x}\right) \\
& D_{y}=\operatorname{path}\left(D_{x}\right)
\end{aligned}
$$

In this way by solving these three nonlinear equations, the three unknowns, $\alpha, \beta$ and $\gamma$ are found and thus the mechanism configuration is determined.

As it was stated before, kinematical analysis of rovers in rough terrains is a cumbersome task. Regarding the shrimp mechanism a nonlinear set of equations should be solved for a position analysis. Since for a velocity or acceleration analysis a derivation process exists and in our case the position terms are not differentiable, a thorough position analysis is required in advance. Then one can use the resultant diagrams to obtain velocity and acceleration with numerical derivation methods.

As it is shown in Fig 2, the shrimp mechanism has 12 linkages. Assuming pure rolling for wheels, 18 two-DOF joints could be considered for this mechanism. According to Kutzbach criterion the system DOF is:

$$
D O F=3(n-1)-2 \times j_{1}-j_{2}=3 \times 12-2 \times 18-0=0
$$

Though the Kutzbach criterion yields zero DOF for this mechanism, it is clear that the right number of DOF is one. This wrong prediction is due to redundant constraint in the middle parallelogram mechanism of the rover. There are several methods to remove this redundancy. Here the cylindrical joint inserting is selected out of available methods. This modification changes the Kutzbach criterion as follows

$$
D O F=3(n-1)-2 \times j_{1}-j_{2}=3 \times 13-2 \times 19-0=1 .
$$




\subsection{Kane's Method}

In this section the inverse dynamical analysis of rovers, using the shrimp rover mechanism as an example is investigated. A dynamical analysis result is a must for objective function derivation, as will be stated in the next section.

Since the optimization procedure consist of several dynamical analyses at each stage, a computationally efficient dynamical analysis is inevitable. In this regard, due to Kane method's merits in complex systems, this method was chosen as the rover dynamical analysis method.

As was discussed in last section the shrimp mechanism's DOF is one. Like Lagrangian mechanics, in which generalized coordinates are employed in rover mechanics, generalized speed is also used to describe the system motion. The number of generalized speeds is equal to the system's DOF; in the case of the shrimp mechanism we need one generalized speed as follows:

$$
u_{1}=\dot{q}_{1}
$$

where $\dot{q}_{1}$ is the rear wheel angular velocity.

The other quantities which have a fundamental role in the construction of generalized forces are partial velocities and partial angular velocities. In order to calculate the partial velocities of a point one should first find the velocities at that point. Then the coefficients of generalized speed in velocity terms are in fact the partial velocities.

Regarding the shrimp mechanism, we have already found the numerical value of velocities. Since we have only one generalized speed in this case; we can obtain the partial velocities and partial angular velocities as follows:

$$
\begin{aligned}
& \mathbf{V}^{\mathbf{P}}=\mathbf{V}^{\mathbf{P}} / u_{1} \\
& \boldsymbol{\omega}^{\mathbf{P}}{ }_{\mathbf{1}}=\boldsymbol{\omega}^{\mathbf{P}} / u_{1}
\end{aligned}
$$

Now, it is possible to get the generalized active and generalized inertial forces. The only external forces and torques are linkage weights and motor torques. Thus for generalized active forces we have:

$$
F_{1}=\sum_{i=1}^{m} \mathbf{M}_{\mathbf{i}} \cdot \boldsymbol{\omega}_{\mathbf{1}}{ }^{\mathbf{W}_{\mathbf{i}}}+m_{i} \mathbf{g} \cdot \mathbf{V}_{\mathbf{1}}^{\mathbf{P}}
$$

where $m_{i}$ represents the mass of different parts of the mechanism and $W_{i}$ represents mechanism wheels; with torque $\mathbf{M}_{\mathbf{i}}$ applied to each.

The generalized inertial force equations are derived as below:

$$
F_{1}^{*}=\sum_{i=1}^{m}\left(-\boldsymbol{\alpha}^{\mathbf{B}_{\mathbf{i}}}\right) \cdot \boldsymbol{\omega}_{\mathbf{1}}{ }^{\mathbf{W}_{\mathbf{i}}}+\left(-\mathbf{a}^{\mathbf{B}_{\mathbf{i}}}\right) \cdot \mathbf{V}_{\mathbf{1}}^{\mathbf{P}}
$$

where $\mathbf{B}_{\mathbf{i}}$ represents different parts of the mechanism which counted as $n$ and $\mathbf{a}^{\mathbf{B}_{\mathbf{i}}}$ is the acceleration of the center of mass of part $\mathbf{B}_{\mathbf{i}}$ and $\boldsymbol{\alpha}^{\mathbf{B}_{\mathbf{i}}}$ is the angular acceleration of 
part $\mathbf{B}_{\mathbf{i}}$. In this way the generalized inertia and generalized active forces are found. Using Eqs. 16 and 17 the following equation of motion is derived:

$$
F_{1}+F_{1}^{*}=0
$$

Which is equivalent to

$$
\sum_{i=1}^{m} \mathbf{M}_{\mathbf{i}} \cdot \boldsymbol{\omega}_{\mathbf{1}}{ }^{\mathbf{W}_{\mathbf{i}}}=\sum_{i=1}^{m}\left(-\boldsymbol{\alpha}^{\mathbf{B}_{\mathbf{i}}}\right) \cdot \boldsymbol{\omega}_{\mathbf{1}}{ }^{\mathbf{W}_{\mathbf{i}}}+\sum_{i=1}^{m}\left(-\mathbf{a}^{\mathbf{B}_{\mathbf{i}}}\right) \cdot \mathbf{V}_{\mathbf{1}}^{\mathbf{P}}-\sum_{i=1}^{m} m_{i} \mathbf{g} \cdot \mathbf{V}_{\mathbf{1}}^{\mathbf{P}}
$$

The right hand side of the Eq. 19 is known, provided we have the accelerations and angular accelerations. Thus an equation for applying torques is obtained. With an appropriate assumed relation between wheel torques, it is possible to calculate each wheel torque. In the shrimp mechanism the following relations are considered between different wheel torques:

$$
\begin{aligned}
& M_{2}=\frac{N_{2}}{N_{1}} M_{1} \\
& M_{3}=\frac{N_{3}}{N_{1}} M_{1} \\
& M_{4}=\frac{N_{4}}{N_{1}} M_{1}
\end{aligned}
$$

where $N_{i}$ is normal force in the wheel-ground interface of $i$ th wheel. This assumption is equivalent with considering more wheel torque as normal force increases. Eqs. 19 and 20 are sufficient for finding wheel torques.

The next step is to calculate the normal forces. Normal forces are among constraint forces and can not be seen in equation of motion; this is expected, as Kane's method is based on energy. Several methods exist to obtain these forces which have been used both in Eqs. 20 and the optimization objective function.

The method introduced in Ref. [12] in order to bring the constraint forces into evident, is to define a set of generalized speeds that violate the constraints. This results in an increase in the numbers of partial velocities and number of governing equations from which the constraint forces and moments are determined. In Ref. [13] the same issue is solved by introducing Lagrange-multiplier-like scalars to adjoin the constraint matrix with Kane's equation for holonomic systems. The resulting equations together with the constraint equations are solved for these scalars and generalized speeds and, hence, for the constraint forces and moments. This approach is suitable for small systems with few degrees of freedom.

Lesser [14] suggested the method of projecting the active and inertia forces and moments on the orthogonal complement space of the configuration space spanned by the partial angular and partial velocities. This requires finding the spanning set of vectors to this defined space and solving the resulting complementary equations for constraint forces and moments.

In this paper the first method was employed for developing the constraint forces (i. e. normal forces). 


\section{Optimization}

To have a rough terrain rover with high mobility that can traverse through unstructured surfaces without loss of wheel-ground contact or slipping, optimization of wheel-ground contact forces is performed. Power efficiency is also considered in rover dynamical optimization. Due to dynamical nonlinearities of the problem, Genetic Algorithm (GA) is selected as the optimization method. GA is a directed random search technique which is applicable to non-linear problems [15].

\subsection{Optimization Criteria}

The most common optimization criterion used for rovers is the minimization of traction in the wheel-ground interface. To avoid wheel slipping, the ratio of traction to normal force in wheel-ground contact point should be lower than a specific value. A function $R_{i}$ that represents this ratio can be used as follows:

$$
R_{i}=\frac{T_{i}}{N_{i}}
$$

where $T_{i}$ is traction and $N_{i}$ is normal force in the wheel-ground interface. $R_{i}$ is also called wheel slip ratio.

For the shrimp rover with four wheels the objective function is selected as the sum of maximum of slip ratios in wheels. Our objective function is in the form of:

$$
O F=\sum_{i=1}^{4} \max \left\{R_{i}\right\}
$$

\subsection{Problem Constraints}

There may be physical constraints for optimization problems. The first one is to keep all wheels in contact with the ground; i.e. normal contact forces should be greater than zero:

$$
N_{i}>0 \quad \text { for } i=1 \ldots 4
$$

The second constraint is that the ratio of traction force to normal force (i.e. $R$ ) should not be greater than the ground-wheel coulomb coefficient of friction:

$$
R_{i} \leq \mu \quad \text { for } i=1 \ldots 4 \text {. }
$$

\section{Simulation Results}

In this section, performance of the optimized shrimp rover and the first version of the rover (i.e. CEDRA-I Shrimp Rover) are compared. Since stairs are challenging terrains, in most cases they are considered as a standard testing rough terrain. In this 
research the terrain is chosen to be standard stairs. Path specifications are listed in Table 1. A sketch of the path and rover are shown in Fig. 3.

CEDRA-I Shrimp Rover is used for the first run. The Rover's specifications including geometric parameters and dynamic properties are listed in Table 2.

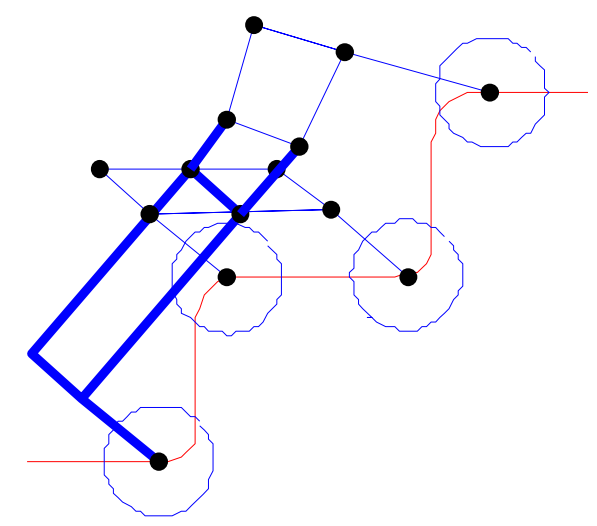

Fig. 3. Sketch of the simulated path and the rover

Table 1. Stairs geometric specifications

\begin{tabular}{|c|c|c|}
\hline $\mathrm{a}$ & $a$ & $0.25 \mathrm{~m}$ \\
\hline $\mathrm{b}$ & $\mathrm{b}$ & $0.25 \mathrm{~m}$ \\
\hline
\end{tabular}

Traversing the stairs path, the rover is dynamically simulated. Dynamic parameters are obtained by solving dynamical equations.

Figure 4 illustrates the results of the simulation. This figure contains rover wheels slip ratio (i.e. $R$ function in Eq. 21). As seen in the figure, the wheel-ground interface slip ratio is close to static coefficient of friction. High slip ratio can reduce the traction at wheel-ground interface; as a result, the rover mobility is reduced.

In the next step, the optimization is applied on the rover. The optimized rover traversed the path and dynamical parameters are obtained by solving dynamical equations obtained using Kane's method. Results containing wheel-ground interface slip ratio are shown in Fig. 5. The geometric parameters of the optimized rover are listed in Table 2.

Obviously the slip ratios are reduced considerably. It can be seen from comparison of Fig. 4 and Fig. 5 that, in some wheels the slip ratio is reduced up to 40 percent. Consequently, the traction at the wheel-ground interface is increased. This is equivalent to the increase in rover mobility that is an important point in rovers. Also it is inspected that there is no loss of contact in wheel-ground interface. 
Table 2. CEDRA-I Shrimp rover and the optimized rover specifications

\begin{tabular}{c|c}
\hline \multicolumn{2}{c}{ CEDRA-I Rover } \\
\hline ITEM & VALUE \\
$\mathrm{b}$ & $0.07 \mathrm{~m}$ \\
$\mathrm{c}$ & $0.29 \mathrm{~m}$ \\
$\mathrm{~d}$ & $0.180 \mathrm{~m}$ \\
$\mathrm{e}$ & $0.130 \mathrm{~m}$ \\
$\mathrm{f}$ & $0.33 \mathrm{~m}$ \\
$\mathrm{~g}$ & $0.01 \mathrm{~m}$ \\
$\mathrm{~h}$ & $0.21 \mathrm{~m}$ \\
$\mathrm{k}$ & $0.21 \mathrm{~m}$ \\
l & $0.16 \mathrm{~m}$ \\
$\mathrm{~m}$ & $0.31 \mathrm{~m}$ \\
$\mathrm{n}$ & $0.21 \mathrm{~m}$ \\
$\mathrm{p}$ & $0.13 \mathrm{~m}$ \\
r1 & $0.05 \mathrm{~m}$ \\
r2 & $0.05 \mathrm{~m}$ \\
r3 & $0.05 \mathrm{~m}$ \\
r4 & $0.05 \mathrm{~m}$ \\
Mass & $40 \mathrm{~kg}$ \\
\hline
\end{tabular}

\begin{tabular}{c|c}
\hline \multicolumn{2}{c}{ Optimized Rover } \\
\hline ITEM & VALUE \\
$\mathrm{b}$ & $0.105 \mathrm{~m}$ \\
$\mathrm{c}$ & $0.277 \mathrm{~m}$ \\
$\mathrm{~d}$ & $0.188 \mathrm{~m}$ \\
$\mathrm{e}$ & $0.116 \mathrm{~m}$ \\
$\mathrm{f}$ & $0.318 \mathrm{~m}$ \\
$\mathrm{~g}$ & $0.035 \mathrm{~m}$ \\
$\mathrm{~h}$ & $0.53 \mathrm{~m}$ \\
$\mathrm{k}$ & $0.284 \mathrm{~m}$ \\
$\mathrm{l}$ & $0.22 \mathrm{~m}$ \\
$\mathrm{~m}$ & $0.26 \mathrm{~m}$ \\
$\mathrm{n}$ & $0.25 \mathrm{~m}$ \\
$\mathrm{p}$ & $0.16 \mathrm{~m}$ \\
r1 & $0.07 \mathrm{~m}$ \\
r2 & $0.07 \mathrm{~m}$ \\
r3 & $0.07 \mathrm{~m}$ \\
r4 & $0.07 \mathrm{~m}$ \\
Mass & $40 \mathrm{~kg}$ \\
\hline
\end{tabular}
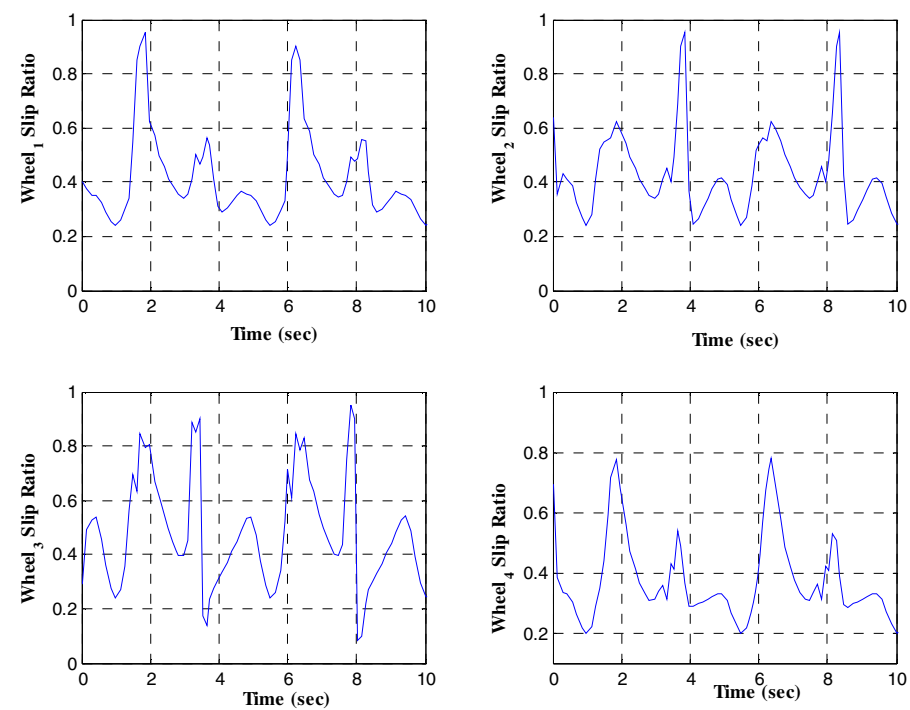

Fig. 4. Slip ratio of wheels for CEDRA-I Shrimp Rover 

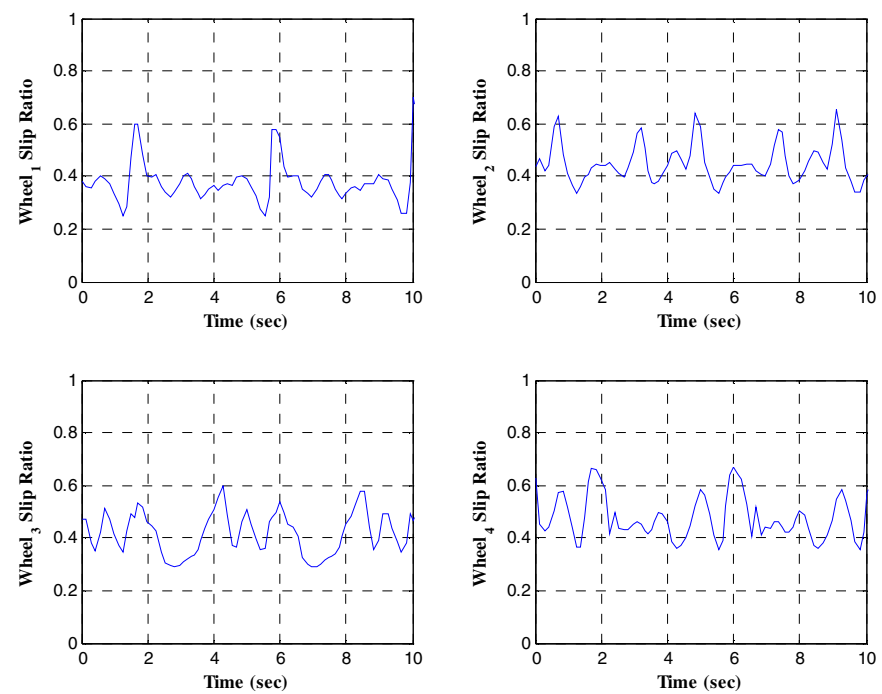

Fig. 5. Slip ratio of wheels for optimized rover

\section{Conclusions}

In this paper an innovative approach to dynamical optimization of rough terrain rovers is presented. Dynamical equations are obtained using Kane's method. Optimization is performed for the rover. Optimization criteria are the minimization of traction in rover wheel-ground interface. Analysis is applied on a 4-wheel rough terrain rover called CEDRA-I Shrimp Rover. Results show an improvement in rover traction, which has an important role in rover mobility.

\section{Acknowledgements}

This project was supported by a grant (82/51065) from the Industrial Development and Renovation Organization of Iran (IDRO). Many thanks are extended to Ms. Ronasi, Dr. Broomand, Dr. Moghaddam, Mr. Ahadi, Mr. Oryani and Ms. Rajabi, for their encouragement and support.

\section{References}

1. Tai M.: Modeling of Wheeled Mobile Robot on Rough Terrain ASME International Mechanical Engineering Congress. Washington D.C. (2003).

2. Iagnemma, K. , and Dubowsky, S.: Mobile Robot Rough-Terrain Control (RTC) for Planetary Exploration. Proceedings of the26th ASME Biennial Mechanisms and Robotics Conference, DETC (2000).

3. Kawabe T., Nakazawa M., Notsu I., Watanabe Y.: Sliding Mode Controller for Wheel Slip Ratio Control System. Journal of Vehicle System Dynamics. 5-6 (1997) 393-408. 
4. Reister, D., Unseren, M.: Position and Constraint Force Control of a Vehicle with Two or More Steerable Drive Wheels. IEEE Transactions on Robotics and Automation. 9 (1993) 723-731.

5. Sreenivasan, S., and Waldron, K.: Displacement Analysis of an Actively Articulated Wheeled Vehicle Configuration with Extensions to Motion Planning on Uneven Terrain. Transactions of the ASME Journal of Mechanical Design. 118 (1996) 312-317.

6. Hacot, H.: Analysis and Traction Control of a Rocker-Bogie Planetary Rover. M.S. Thesis, Massachusetts Institute of Technology. Cambridge, MA (1998).

7. Meghdari A., Pishkenari H. N., Gaskarimahalle A. L., Mahboobi S. H., Karimi R.: Optimal Design and Fabrication of CEDRA Rescue Robot Using Genetic Algorithm. Proc. of the International Design Engineering Technical Conferences DETC. Salt Lake City, Utah (2004).

8. Kane T. R., Levinson D. A.: The use of Kane's Dynamical Equations in Robotics. Int. J. Robotics Research. 7 (1996) 333-342.

9. Estier T., Crausaz Y., Merminod B., Lauria M., Piguet R., Siegwart R.: An Innovative Space Rover with Extended Climbing Abilities. In Proceedings of Space \& Robotics, the Fourth International Conference and Exposition on Robotics in Challenging Environments. Albuquerque, New Mexico (2000).

10. Volpe R., Balaram J., Ohm T., Ivlev R..: Rocky 7: A Next Generation Mars Rover Prototype. Journal of Advanced Robotics. 4 (1997).

11. Kemurdjian, A. L., Gromov, V., Mishkinyuk, V., Kucherenko, Sologub, P.: Small Marsokhod Configuration. International Conference on Robotics \& Automation. Nice (1992).

12. Kane T. R., and Levinson D. A.: Dynamics: Theory and Applications. 1st edn. Series in Mechanical Engineering, McGraw-Hill, New York (1985).

13. Wang J. T., and Huston R. L.: Kane's Equations with Undetermined MultipliersApplication to Constrained Multibody Systems. Journal of Applies Mechanics. 2 (1987) 424-429.

14. Lesser M.: A Geometrical Interpretayion of Kane's Equations. Proceedings of the Royal Society of London, Series A: Mathematical and Physiscal Sciences. 1896 (1992) 69-87.

15. D.T. Pham, D. Karaboga: Intelligent Optimization Techniques. Springer-Verlag, New York (2000). 